\title{
Aging test of high rate MRPC
}

\section{Yi Wang'}

Department of Engineering Physics, Tsinghua University, Key Laboratory of Particle \& Radiation Imaging, Ministry of Education

Beijing 100084, China

E-mail: yiwang@mail.tsinghua.edu.cn

\section{Xingming Fan ${ }^{\mathrm{a}}$, Huangshan Chen ${ }^{\mathrm{a}}$, Jingbo Wang ${ }^{\mathrm{a}}$, Diego Gonzalez-Diaz ${ }^{\mathrm{a}, \mathrm{b}, \mathrm{c}}$, Yuanjing $\mathrm{Li}^{\mathrm{a}}$, Jianping Cheng ${ }^{\mathrm{a}}$}

${ }^{a}$ Department of Engineering Physics, Tsinghua University, Key Laboratory of Particle \& Radiation Imaging, Ministry of Education

Beijing 100084, China

${ }^{b}$ GSI Helmholtz Center for Heavy Ion Research, Darmstadt, Germany

${ }^{c}$ Laboratorio de Física Nuclear y Altas Energías, Universidad de Zaragoza, Zaragoza, Spain

A new kind of low resistivity glass has been developed. Its volume resistivity is on the order of $10^{10} \Omega \mathrm{cm}$ and MRPCs assembled with it show a very promising rate capability $\left(>25 \mathrm{kHz} / \mathrm{cm}^{2}\right)$. This new detector has a very important range of application in high energy physics experiments such as FAIR-CBM, LHC-ATLAS or Jlab-SOLID, to mention some. In this paper we report new results related to its long-term behavior (ageing). The module has been irradiated by X-rays at a mips-equivalent flux of $15 \mathrm{kHz} / \mathrm{cm}^{2}$, for 300 hours and for a released charge totaling $0.22 \mathrm{C}$. No noticeable degradation could be observed. Compared to common glass MRPCs, the newly developed high rate counter responds faster to sudden irradiation.

XI workshop on Resistive Plate Chambers and Related Detectors (RPC2012)

INFN-Laboratori Nazionali di Frascati, Italy

February 5-10, 2012

$1 \quad$ Yi wang, yiwang@mail.tsinghua.edu.cn 


\section{Introduction}

The Compressed Baryonic Matter (CBM) experiment, proposed at the future Facility for Antiproton and Ion Research (FAIR) in Darmstadt, has decided to use the MRPC technology to build its Time-Of-Flight (TOF) wall[1]. Compared to other experiments (for instance ALICE, STAR, et al), CBM-TOF requires a rate capability as high as 20 $\mathrm{kHz} / \mathrm{cm}^{2}$. Tsinghua University is a group member of CBM-TOF and is doing research on low-resistivity glass and high rate MRPC. The volume resistivity of our glass is on the order of $10^{10} \Omega \mathrm{cm}$. High rate MRPCs preserve an excellent 50ps intrinsic time resolution under a load of as much as $35 \mathrm{kHz} / \mathrm{cm}^{2}$ of minimum ionizing particles [2].

In this paper we report results of an aging test conducted on high rate MRPCs. One module with $100.22 \mathrm{~mm}$-gap and 12 readout pads $(2 \mathrm{~cm} \mathrm{x} 2 \mathrm{~cm})$ was tested with a $60 \mathrm{kV}$ $\mathrm{X}$-ray source. By Monte Carlo simulations, we estimate that characteristic energy distribution of bremsstrahlung X-rays is rather penetrating as to consider irradiation to be fairly uniform for all the gaps, within a maximum variation of a factor $\mathrm{x} 2$.

\section{Experimental setup and data taking}

The experiment was done with the high rate pad MRPC[3]. It was assembled with low resistivity glass. The dimension of the readout pad is $2 \mathrm{~cm} \times 2 \mathrm{~cm}$. The detector consists of ten gaps and the gap width is $0.22 \mathrm{~mm}$. The working gas consists of $90 \%$ Freon, $5 \%$ iso-butane and $5 \% \mathrm{SF}_{6}$. The working voltage is $12 \mathrm{kV}$. An $\mathrm{X}$ ray machine (Spellman $\left.{ }^{\mathrm{TM}} \mathrm{XRB} 80\right)$ was used to do the radiation experiment and irradiation performed perpendicularly to the RPC plates. In order to monitor the performance, two digital rate meter were used to record the current and signal rate per minute. The monitoring system is shown in Fig.1. The current of the MRPC can be obtained from the HV source (CAEN, N471) and the counting rate was obtained from the output of the Front End Electronics[4].

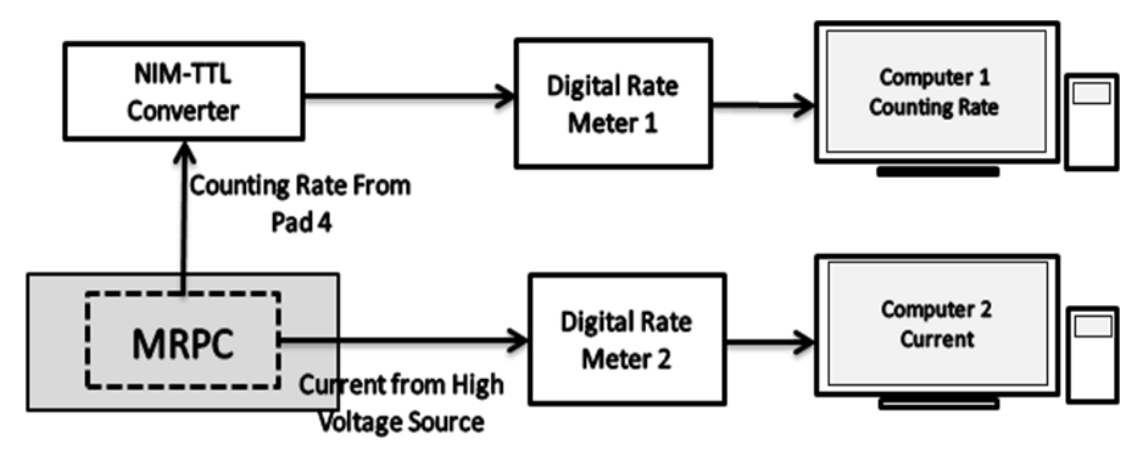

Fig.1 Schematic diagram of the monitoring system.

The dose rateis equal to be irradiated with $30 \mathrm{MeV}$ electron with flux rate of $15 \mathrm{kHz} / \mathrm{cm}^{2}$. This can be seen in Fig.2. There is a very good linearity between the current and the electron flux. When the electron flux is $15 \mathrm{kHz} / \mathrm{cm}^{2}$, the current is about $50 \mathrm{nA} / \mathrm{cm}^{2}$ that corresponds to some $1.7 \mathrm{pC}$ /avalanche (the structure is mirrored). A lead collimator with 
a $4 \mathrm{~cm}^{2}$ hole was put in front of the gas box, thus fixing the irradiated area to that size. Therefore, the observed working currents of $200 \mathrm{nA}$, roughly correspond to a $30 \mathrm{MeV}$ electron flux of $15 \mathrm{kHz} / \mathrm{cm}^{2}$ (that we operationally assimilate to minimum ionizing particles in the following). The temperature and moisture were kept constant during the test.

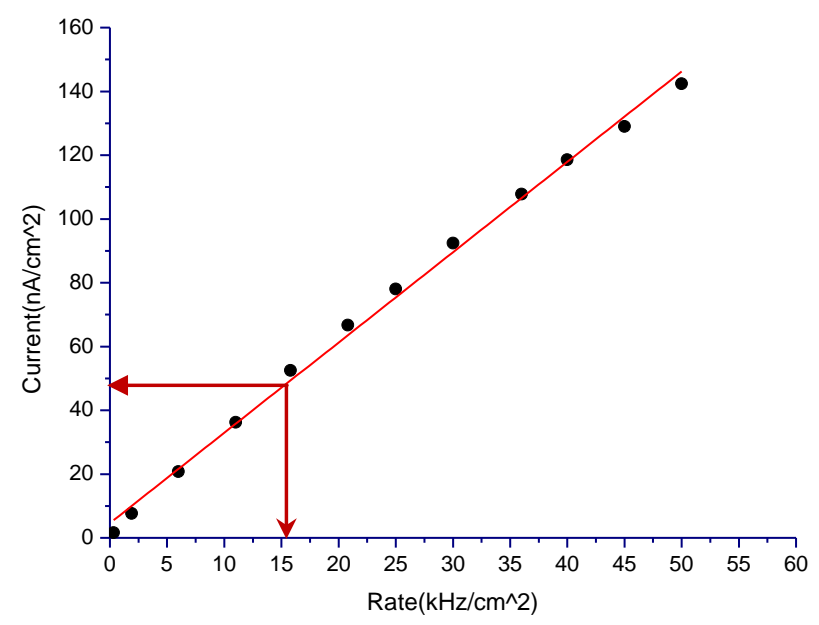

Fig.2 The current of the pad-readout MRPC changing with respect to the electron flux during the HZDR beam test (30MeV electrons). For a mirrored RPC this almost linear relation corresponds to $1.7 \mathrm{pC} /$ avalanche. A detailed look reveals that the growth is less-than-proportional at the highest fluxes, as expected.

Since the MRPC consists of 12 pieces of $0.7 \mathrm{~mm}$-thick glasses, which may cause scattering and attenuation of incident photons, we simulated the photons transport across the MRPC with MCNP-4C. The working gas is not expected to be sensitive to the incident photons and virtually any avalanche will be started by the secondary electrons generated by photon interactions in the glass. For $60 \mathrm{keV} \mathrm{X}$-rays, simulations show that secondary electrons stem both from photo-electric effect and from Compton scattering. Fig. 3 shows the number of electrons produced in each gas gap. From 1 to 2.5 electrons can be produced in the gas gap per 100 incident photons. The detector consists of two stacks, and the electron number decreased by about $30 \%$ in each stack due to the attenuation of the X-ray beam. We expect the multi-gap equilibration mechanism can cope with this slight unbalance by a proportional increase in the avalanche charge of the less irradiated gaps. Overall, we expect a factor $\mathrm{x} 2$ decrease in the particle load from the first gap up-stream to the latest. Although a detailed extrapolation to (fully penetrating) minimum ionizing particles is not straight, we can safely assume a systematic error of a factor of two for all ageing figures given here, that is a reasonable value for this kind of tests. 


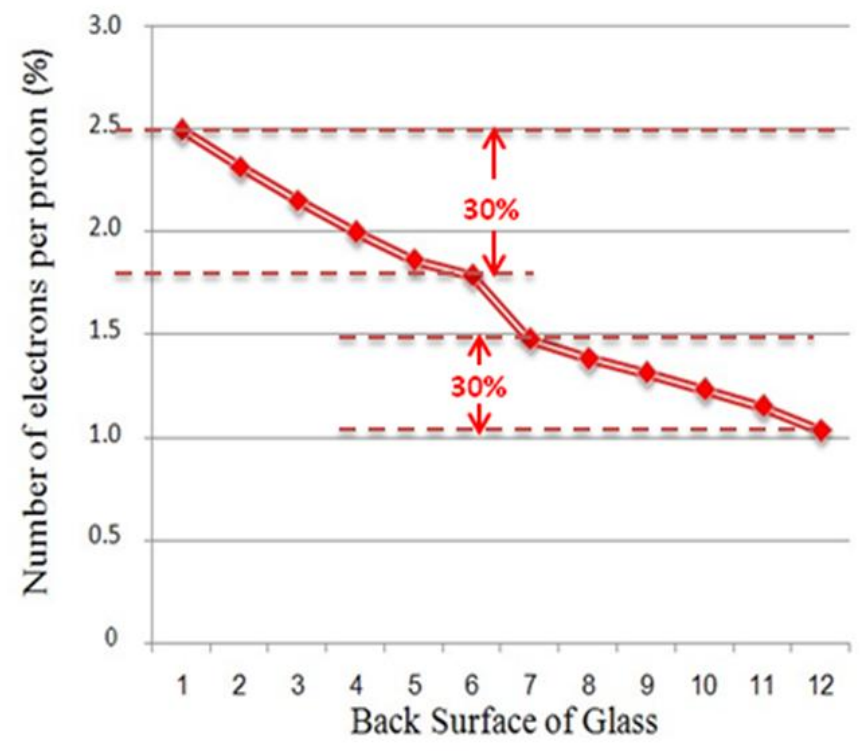

Fig.3 The number of electrons produced in each gas gap according to the Monte Carlo simulator MCNP-4C.

\section{Experimental results and discussion}

The aging test is divided in three stages, each stage lasting for approximately 100 hours. Fig. 4 shows the counting rate and current change with test time during the first stage. To protect the $\mathrm{X}$ ray supply, the machine had to be switched off for 30 minutes every 8 hours of irradiation (no auxiliary cooling was implemented) while on Fig.4 there are several troughs as corresponding. It can be seen that the current stayed at $200 \mathrm{nA}$ and the counting rate was flat at around $35 \mathrm{kHz} / \mathrm{pad}$, Which is expected to roughly correspond to $15 \mathrm{kHz} / \mathrm{cm}^{2}$ electron flux irradiation. The counting rate and current are very stable during these 100 hours irradiation. The performance is nearly the same in the next two stages. Between each stage, the MRPC was tested with cosmic rays. Efficiency, time resolution and other performances were tested. The charge spectrum before and after irradiation is shown in Fig.5, where no substantial change can be observed. The time resolution of the 4th pad is $90 \mathrm{ps}$ before irradiation, it becomes 95ps after 100 hours irradiation and it is about 90ps after 300 hours irradiation. The efficiency, dark current and noise rate are nearly the same as before irradiation. In conclusion, we do not observe any significant performance degradation for this detector. 


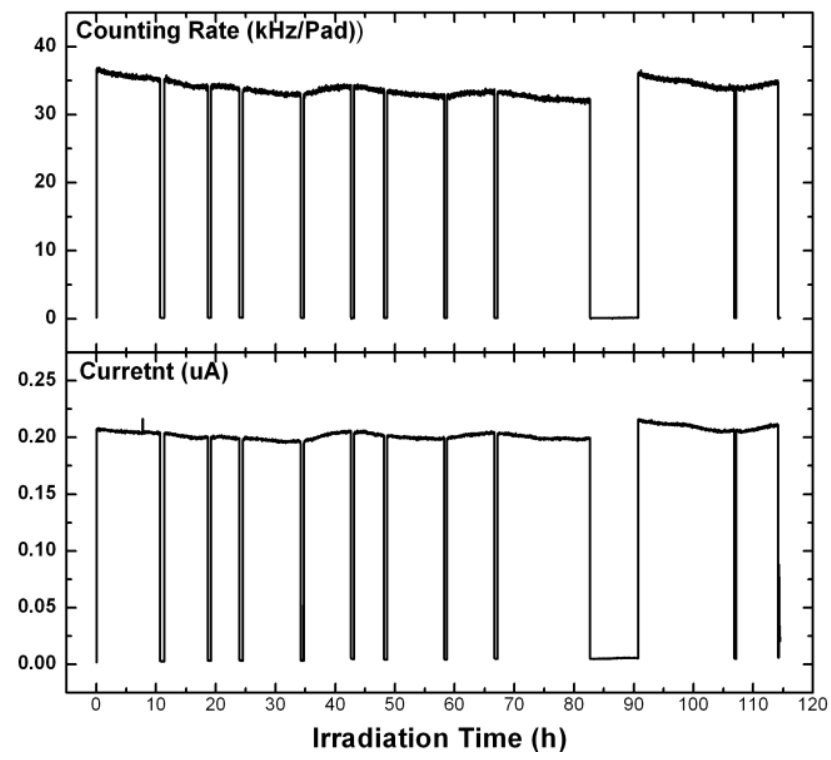

Fig.4 Counting rate and current with respect to time during the first stage.

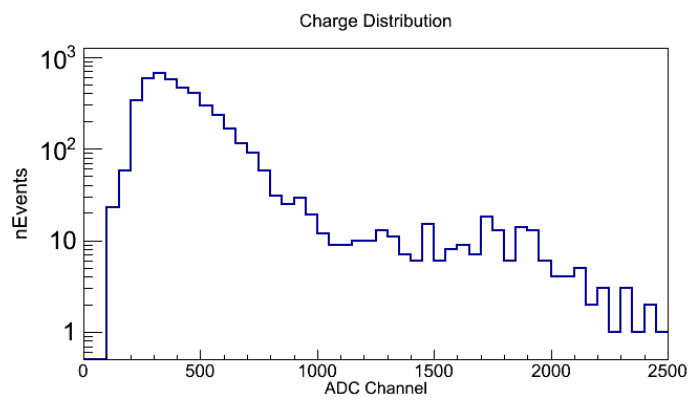

Fig.5(a)

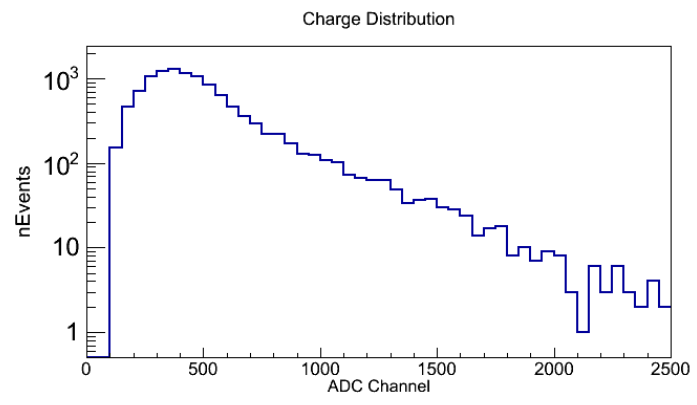

Fig.5(b)

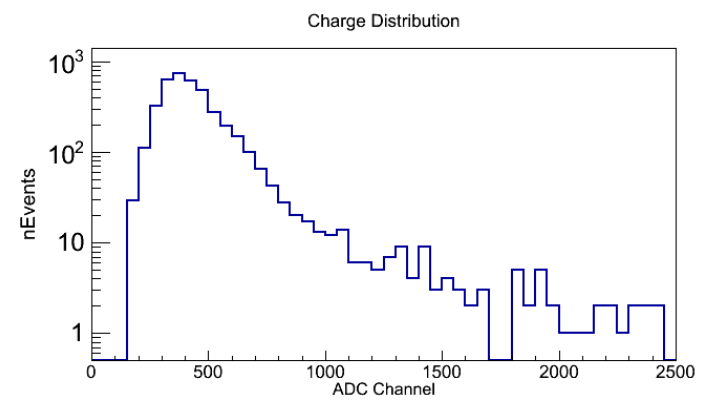

Fig.5(c)

Fig.5 Charge distribution of the MRPC before and after different periods of irradiation:

a) Charge distribution before irradiation.

b) Charge distribution after 100 hours of irradiation.

c) Charge distribution after 300 hours of irradiation 


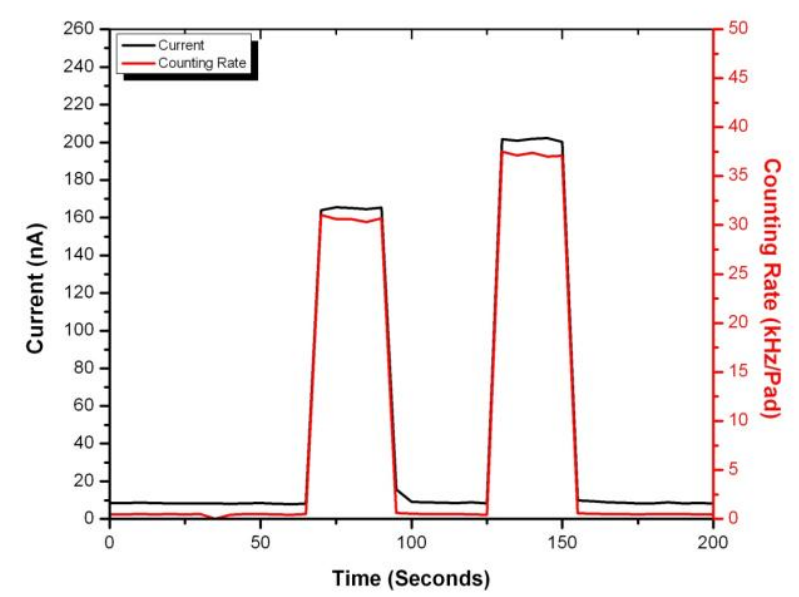

Fig.6 Transient response to irradiation of the high rate MRPC.

The MRPC assembled with low resistive glass can afford a high particle flux, and it also responds to irradiation faster than the MRPC assembled with common window glass, as expected. Fig. 6 and 7 show the comparison between high rate MRPC and common glass MRPC. When the X-ray supply is turned on, for the high rate MRPC, the current takes a few seconds to reach a stable state. For common glass MRPC, at much lower fluxes, the process can last few hours in some circumstances. The current and counting rate might go first up and then decrease until they gradually stabilize[5]. For high rate MRPC, these performances reach a stable state immediately. We note that for irradiation at milder particle fluxes, as in [6], the observed transient time of common glass is just few seconds, in agreement with the expectation from its measured AC response.

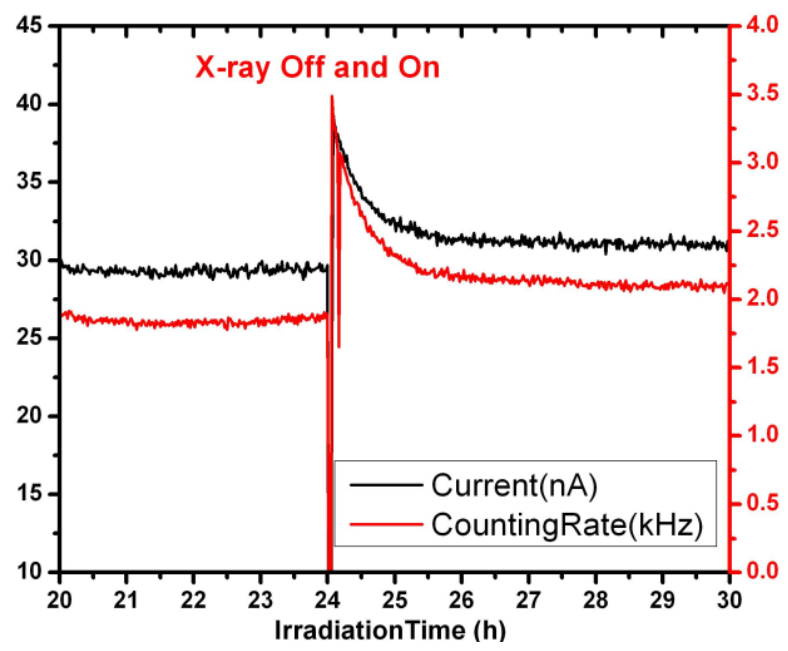

Fig.7 Transient response to irradiation of a common glass MRPC. 


\section{Conclusions}

We started tests aimed at studying the performance of high rate MRPC under long term irradiation. The module has been irradiated by X-rays for 300 hours and the accumulated charge reached $0.2 \mathrm{C}\left(0.05 \mathrm{C} / \mathrm{cm}^{2}\right)$, already exceeding the practical operating life of standard float glass RPCs. The efficiency, time resolution and noise rate were tested every 100 hours of irradiation in order to check its performance degradation. The results show that there is indeed no significant performance degradation. Interestingly, the high rate MRPC responds very fast to sudden changes of the irradiation conditions (e.g. on and off). Detailed tests are still being done to ensure the long time stable running of high rate MRPCs up to much longer densities of transported charge.

\section{Acknowledgements}

This work is supported by the National Natural Science Foundation of China under Grant No.s 11020101059, 10775082, 111050110573 and 10979030, it is also supported by Tsinghua University Initiative Scientific Research Program.

\section{References}

[1] I. Deppner, et al.,The CBM time-of-flight wall. Nucl. Instr. and Meth. A661(2012) S121-S124.

[2] Jingbo Wang, Yi Wang, et al. Development of multi-gap resistive plate chambers with lowresistive silicate glass electrodes for operation at high particle fluxes and large transported charges. Nucl. Instr. and Meth. A 621 (2010) 151.

[3] Yi Wang et al. Progress of R\&D and production of timing RPCs in Tsinghua University. Nucl. Instr. Meth. A 661 (2012) S134-S136.

[4] F. Geurts, et al., Nucl. Instr. and Meth. A 533 (2004) 60.

[5] Yi Wang et al. Aging test of multi-gap resistive plate chambers. Chinese Physics C, 2006(30), 441-444.

[6] D. Gonzalez-Diaz et al., Nucl. Instr. Meth. A, 602(2009)713. 